\title{
RFID-Technologie : Bindeglied zwischen Informationsfluss und Materialfluss. Wie zuverlässig sind die Identifikationsvorgänge?
}

\author{
PROF. KARL-HEINZ WEHKING \\ DIPL.-ING. FRANK SEEGER \\ DIPL-ING. STEPHAN KUMMER \\ UNIVERSITÄT STUTTGART, INSTITUT FÜR FÖRDERTECHNIK UND LOGISTIK (IFT)
}

\section{Einleitung}

Mit der zunehmenden Komplexität der Logistikketten ist auch der Anspruch an die Informationstransparenz gestiegen. Hier ergibt sich durch den Einsatz intelligenter RFID -Technologie eine weitere Möglichkeit zur Optimierung und Steuerung der eingesetzten Kapazitäten, denn so ist eine Identifikation und Verfolgung von Waren und Gütern entlang der gesamten Supply Chain möglich. Jedes Produkt ist zu jeder Zeit genau lokalisierbar und kann eine Fülle an aktuellen und historischen Daten liefern.

Durch den Einsatz der RFID-Technologie kann das Zusammenspiel von Materialfluss und Informationsfluss zwischen den einzelnen Prozessschritten optimiert werden. Damit ist gewährleistet, dass alle erforderlichen Daten zum richtigen Zeitpunkt am richtigen Ort sind. Die dezentrale Bereitstellung und Veränderung von Daten ermöglicht eine flexible, dezentrale Steuerung logistischer Systeme. Weitere Vorteile der RFID - Komponenten sind beispielsweise, dass sie individuell beschreibbar sind und ihre Identifikation selbst über begrenzte Entfernungen und ohne Sichtkontakt zwischen Tag und Lesegerät möglich ist.

Durch den Einsatz von RFID-Transpondern eröffnen sich Potentiale in Richtung Prozesssicherheit, Reduzierung der Logistikkosten oder auch Verfügbarkeit der Produkte.

Diese Vorteile hängen jedoch nicht zuletzt von der Zuverlässigkeit der Identifikationsvorgänge ab. Die unbestrittenen Potentiale, die durch Einsatz von RFID-Elementen erschlossen werden können, sind nur dann nutzbar, wenn die nun dezentral an den Waren oder Ladeeinheiten zugeordneten Informationen an den benötigten Stellen zuverlässig abgerufen werden können. Die Kommunikation zwischen Transponder und Lese/Schreibeinrichtung wird durch unterschiedliche Materialen, wie z.B. Metall, die die Funkverbindung beeinflussen erschwert. Die Zuverlässigkeit dieser Kommunikation wird anhand von Versuchen, mit unterschiedlichen Füllmaterialien und unterschiedlichen Anordnungen der Transponder an den Ladeeinheiten, ermittelt.

\section{Grundlagen der RFID-Technologie}

Der Begriff RFID bedeutet Radio-Frequency-IDentification und beschreibt das Verfahren zur Kommunikation zwischen Transponder und der entsprechenden Schreib-/ Lesestation.

Gegenüber der Identifikation mit dem heute üblichen Barcode Verfahren bietet der Einsatz von RFID- Technik folgende Vorteile [Overmeyer05]:

- Zusätzliche Informationen können direkt auf dem Transponder hinterlegt werden (z.B. Haltbarkeitsdatum, Herkunft,...)

- Je nach Systemausführung können die Transponder innerhalb des logistischen Systems beschrieben werden

- Eindeutige Identifikation der Ware mittels der Ein-Eindeutigen ID-Nummer des Transponders

- Zwischen Transponder und Lesestation ist keine Sichtverbindung nötig

- Identifikation ist ohne zusätzliches Handhaben der Ware möglich

- Transponder können geschützt vor äußeren mechanischen (Beschädigung, Verlust,...) oder chemischen (Säuren, Schmutz,...) Einflüssen angebracht werden

- Erkennung mehrerer Transponder während eines Lesevorganges möglich (Pulkerfassung)

Transponder - äquivalent wird auch der englische Begriff „Tag“ verwendet - ist ein Kunstwort und besteht aus den beiden Wortteilen Transmitter (to transmit: senden) und Responder (to respond: antworten). Zu einem RFID- System gehören immer zwei Komponenten: die Schreib-/Lese- Einrichtung mit dazugehöriger Auswerteeinheit [Finkenzeller02], [Bapat04] zur Verwaltung der Daten sowie mindestens ein Transponder.

Bei RFID-Anlage spielt die Betriebsfrequenz eine entscheidende Rolle. Die Frequenz des Systems hat maßgeblichen Einfluss auf die Reichweite und das Verhalten in verschiedenen Medien. Vereinfachend kann man aber sagen, dass mit steigender Frequenz zum einen die benötigte Betriebsleistung sinkt und sich zum anderen die theoretische Reichweite und Datenübertragungsrate erhöht. Allerdings ändert sich auch das Reflexions- bzw. das Absorbtionsverhalten des Feldes bei Auftreffen auf verschiedene Materialien wie z.B. Metall oder Wasser.

Rechtlich betrachtet handelt es sich bei RFID-Systemen um Funkanlagen. Diese unterliegen daher den entsprechenden gesetzlichen Bestimmungen. Somit bleiben unter Beachtung anderer existierender Funkbetreiber 
und Nutzer nur wenige freie Frequenzbänder übrig, von denen hauptsächlich folgende für RFID-Zwecke genutzt werden:

- Niederfrequenz (NF):

- Hochfrequenz (HF):

- Ultrahochfrequenz (UHF):

- Mikrowelle (MW):
$135 \mathrm{KHz}$

$13,56 \mathrm{MHz}$

$868 \mathrm{MHz}$ (Europa) bzw. $915 \mathrm{MHz}$ (USA)

$2,45 \mathrm{GHz}$

Des Weiteren können Transponder nach Art ihrer Stromversorgung unterschieden werden:

- Passive Transponder: Der Transponder gewinnt seine für den Betrieb notwendige Energie durch die Einkopplung in das Antennenfeld. Er besitzt keine eigene Energiequelle.

- Aktive Transponder: Sie werden durch eine aktive Energiequelle (Batterie) gespeist, d.h. sie benötigen für das Senden ihrer Daten nicht die Energie aus dem Antennenfeld. Im Gegensatz zu passiven Transpondern sind sie teurer, besitzen allerdings auch eine vielfach höhere Sendeleistung.

- Semiaktive Transponder: Diese besitzen auch eine aktive Energiequelle, die allerdings für den Betrieb einer dort implementierten Zusatzfunktion in Form unterschiedlicher Messsensoren Verwendung findet (z.B. Temperaturüberwachung). Der eigentliche Transponder bezieht seine benötigte Energie wie ein passiver aus dem Antennenfeld.

\section{Standardisierung}

Damit RFID auch firmenübergreifend in der gesamten logistischen Kette [tenHompel04], [Friess00] mit mehreren Partnern reibungsfrei funktioniert, ist eine Standardisierung und Normung sowohl auf Seiten der Hardware als auch der Software bzw. Datenstrukturen notwendig. Dieser für den weltweiten Einsatz von RFID nötige Standardisierungsprozess [Roos02] ist auch auf lange Sicht noch nicht abgeschlossen.

Standardisierungsbestrebungen gibt es von mehreren unabhängigen Organisationen. Die Interessen von Handel, Konsumgüterindustrie sowie Technologielieferanten und Beratern werden beispielsweise von der EPC Global vertreten. Sie wird von der GS-1 Germany (vormals Centrale für Coorganisation (CCG) ) [GS1o.J] sowie dem Uniform Code Council (UCC) getragen. Seitens der Automobilindustrie bündelt der VDA die Normungsund Standardisierungsbestrebungen.

Die jeweiligen Standardisierungsbemühungen und Regelwerke [Jansen05] können nach den von Ihnen fokussierten Teilaspekten untergliedert werden:

\section{Technologiestandards:}

Hier werden die grundlegenden technischen Eigenschaften der RFID-Systeme beschrieben und festgelegt, z.B. die zu verwendenden Frequenzbereiche und die Ausgestaltung von kontaktlosen Chipkarten. Erwähnenswert sind auch mehrere Normen, wie z.B. die ISO 18000, die sich mit der Luftschnittstelle Antenne - Transponder befasst.

\section{Anwendungsstandards:}

Sie empfehlen auf einzelne Anwendungen angepasste technische Lösungen. Außer im Bereich der Tieridentifikation im Niederfrequenzbereich (ISO 11784, 11785 und 14223) und im Bereich der Containeridentifikation mit aktiven UHF- und MW- Systemen (ISO 10374 und 23389) sind Anwendungsstandards bis heute eher rar. In Arbeit sind zurzeit noch die ISO-Normen 17363 und 17367, die alle Bündelungsebenen in einem logistischen System betrachten.

\section{Datenstandards:}

Hier geht es um die Standardisierung von Datenformaten und der Datenorganisation, damit ein reibungsfreier Datenaustausch aller benötigter Daten innerhalb der Supply-Chain möglich ist. Als Beispiel können die in ISO 15963 beschriebene Identifikationsnummer oder auch das in ISO 15962 behandelte Datenprotokoll von Schreib/Leseeinheiten genannt werden.

\section{Zulassungsvorschriften:}

RFID- Systeme gelten, wie bereits erwähnt, als Funkanlagen und unterliegen dementsprechenden Vorschriften. Die EN 300330 ist für NF- und HF-Anwendungen zu beachten, die EN 300440 für den MW-Bereich. Für den UHF-Bereich gilt die EN 300220, welche zurzeit noch eine Begrenzung der Sendeleistung von 0,5 Watt vorschreibt. In den USA liegt dieser Wert bei 4 Watt. Eine Anhebung der Sendeleistung von 868 MHz RFIDLesegeräten in Europa auf 2 Watt ermöglicht die kürzlich vom European Telecomunications Standards Institute (ETSI) verabschiedete EN 302208.

\section{Schwachstelle - Zuverlässigkeit des Schreib-Lese-Zugriffs}

Der Transponder ist ein wichtiger Bestandteil für die Sicherstellung des Informationsflusses in logistischen Systemen. Die Vorteile des RFID-Einsatzes hängen aber nicht zuletzt von der Zuverlässigkeit und Verfügbarkeit @2006 by Logistics Journal : Referierte Veröffentlichungen - ISSN 1860-7977 
der Identifikationsvorgänge ab. Die Potentiale, die durch die Implementierung von RFID-Systemen erschlossen werden können, sind nur dann voll nutzbar, wenn die nun dezentral direkt den Waren oder Ladeeinheiten zugeordneten Informationen an den benötigten Stellen zuverlässig abgerufen werden können.

Werden die Informationen bei Übergängen zwischen den Schnittstellen von logistischen Prozessen nicht zuverlässig weitergegeben, z.B. bei der Bündelung von Einzelgütern zu Kommissionen, entsteht im Informationsfluss ein Bruch im Netz. Reißt dieser Informationsfluss ab, können die Potentiale der RFID-Technik nicht genutzt werden und Back-up Lösungen müssten in diesem Fall einspringen (z.B. konventioneller Lieferschein, Abgleich mit Datenbank).

Die Kommunikationssicherheit zwischen Transponder und Schreib-/Lesestation ist von vielen Faktoren abhängig, die bei der Implementierung von RFID Beachtung finden müssen:

- $\quad$ Systemfrequenz

- Datenmenge

- Art und Anzahl der Transponder im Lesefeld

- Ausrichtung und Position der Transponder untereinander

- Ausrichtung und Position im Antennenfeld

- Art, Menge und Verteilung kritischer Materialien wie Metall oder Wasser am belabelten Objekt

\section{Versuchsstand zur Pulk-Erfassung an KLTs}

Das IFT führt zurzeit Untersuchungen zur Pulkerfassung von passiven 13,56 MHz-Transpondern durch. Ziel dieser Versuche ist die Ermittlung der Erkennungszuverlässigkeit von Transpondern und der entsprechenden Ladeeinheiten unter realitätsnahen Bedingungen industrieller Anwendungen. Es werden hierbei die Einflüsse der Transponderposition und unterschiedlicher Füllstoffe auf das Erkennungsverhalten berücksichtigt.

Zur Durchführung der Versuche steht eine Förderstrecke, bestehend aus Rollen- und Kettenförderern mit einer Tragkraft von 250 kg zur Verfügung (s. Abb. 1). Die Palette wird reversierend mit einer Geschwindigkeit von ca. $0,5 \mathrm{~m} / \mathrm{s}$ über die Rollenförderstrecke gefördert, in deren Mitte die RFID-Anlage angebracht ist. Es handelt sich hierbei um eine zweiseitige Gate-Antenne von Texas-Instruments für den 13,56 MHz-Bereich.

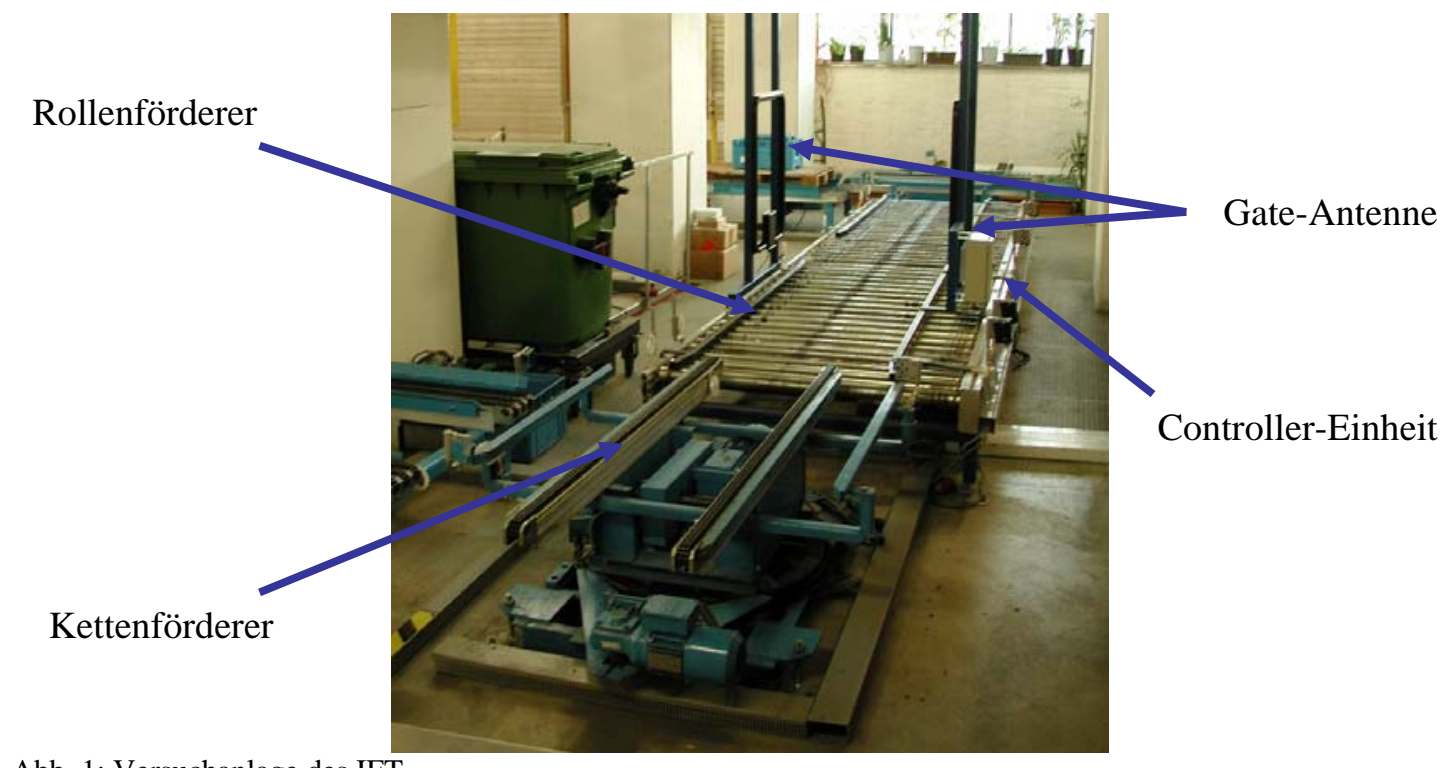

Abb. 1: Versuchanlage des IFT

\subsection{Versuchsaufbau}

Für die Versuchsdurchführung werden zwei Versuchsaufbauten mit den Transponderanordnungen Variante A und Variante B verwendet. Bei beiden Versuchsaufbauten wird als Grundladungsträger eine Euro-Palette benutzt, auf die jeweils 12 C-KLT 6428 (Maße L*B*H: 600*400*280 mm) in drei Lagen aufgestapelt werden. In der Automobilindustrie stellen diese Kleinladungsträger (KLT) mit mittlerweile über 35 Millionen Exemplaren den branchenweiten Standardbehälter dar. Auf jedem KLT befinden sich vier passive Transponder (48 je Palette), sog. Smart-Label; also je KLT-Seite einer. Dieser Aufbau hat sich aus Vorversuchen, die später noch beschrieben werden, ergeben. Der Unterschied besteht in der Positionierung der Transponder auf den Seitenflächen. 

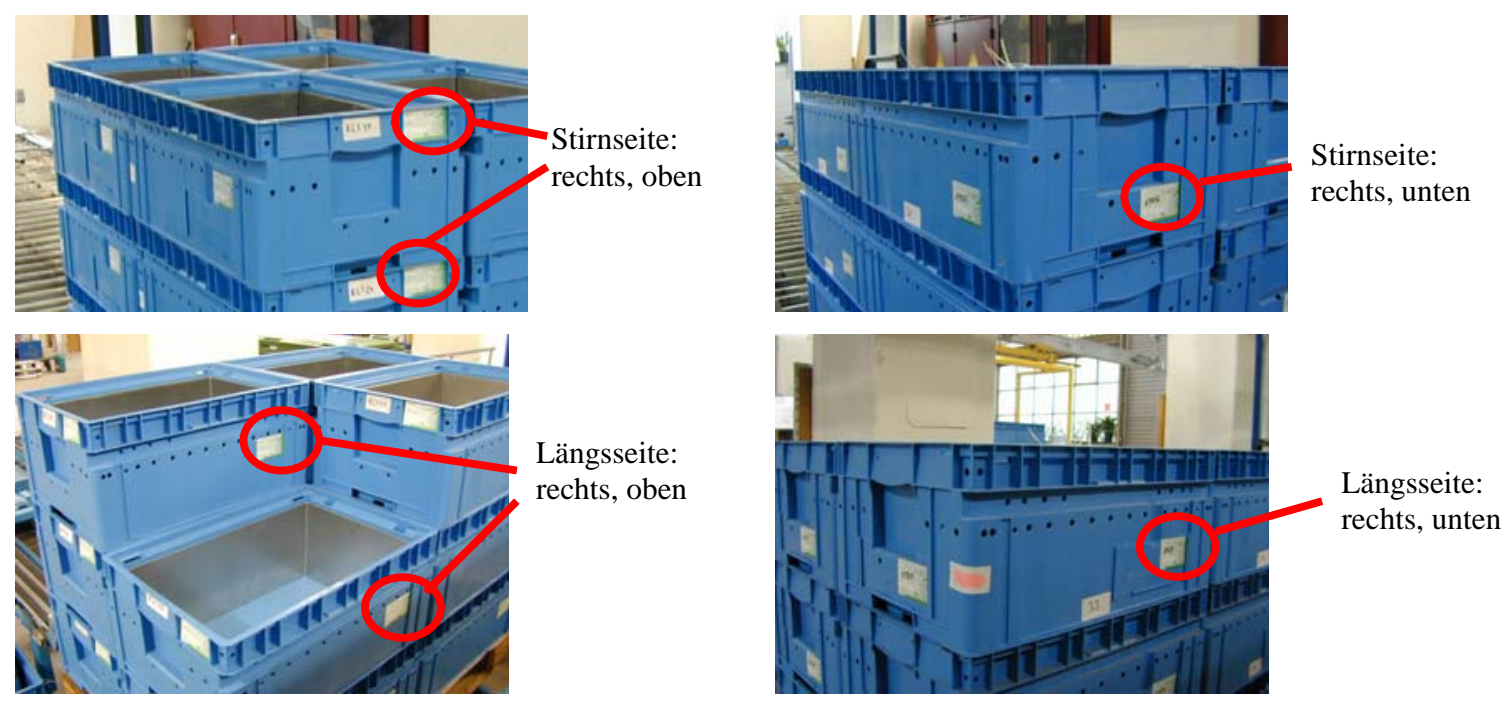

Transponderanordnung Variante A

Transponderanordnung Variante B

Abb. 2: Vergleich der Transponderanordnung zwischen Aufbauvariante A und B

In Abbildung 2 sind beide Aufbauvarianten zu sehen. Bei Variante A sind die stirnseitigen Transponder jeweils rechts oben angebracht. Die Transponder der Längsseiten befinden sich unterhalb der dort angebrachten Lüftungsöffnungen. Die Transponder bei Variante B sind, wie in Abbildung 3 zu sehen, insgesamt tiefer angeordnet.

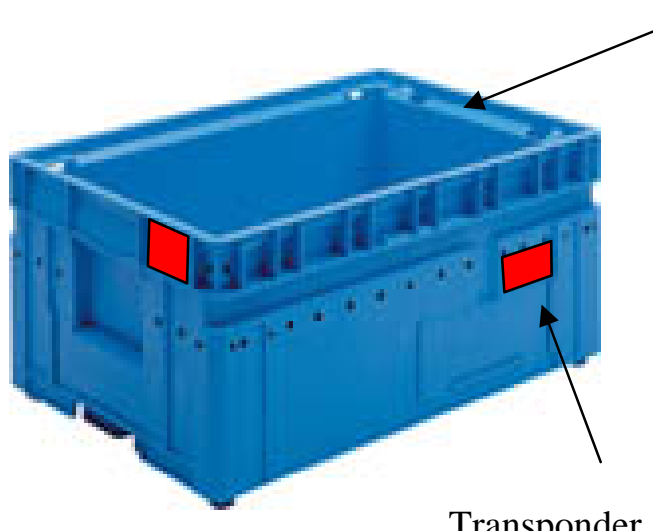

Transponderanordnung Variante A

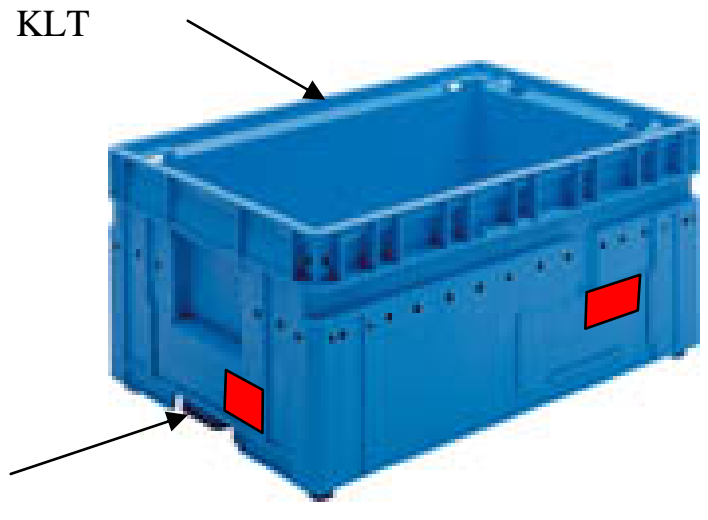

Transponderanordnung Variante B

Abb. 3: Transponderanordnung Variante A und Variante B

Bei einer Stapelung der KLT ergibt sich, bedingt durch ein leichtes Eintauchen der KLT ineinander (Vertiefung des Bodens, setzt auf den Innenrand des unteren Behälters auf), dass die theoretische Behälterhöhe nicht komplett genutzt werden kann. Hierdurch entstehen zwischen den einzelnen Stapellagen Spalte, die nicht direkt von der Versuchsbeladung abgeschirmt sind. Um den Einfluss solcher nicht vermeidbarer „Spalte“ auf die Lesbarkeit von Transponder zu überprüfen, wurden die stirnseitigen Transponder bei Variante A möglichst hoch platziert, so dass sie innerhalb dieser Spalte liegen. Bei Variante B werden die Transponder voll von den Füllstoffen „abgeschirmt“.

\subsection{Inbetriebnahme der Anlage}

Die verwendete Gate-Antenne besteht aus einer Basis- und einer Ergänzungsantenne, die beiderseits der Versuchsförderstrecke (s. Abb. 1), einem Rollenförderer, montiert worden sind. Beide Antennenteile wurden jeweils im gleichen Abstand senkrecht stehend, parallel zur Förderstrecke aufgebaut. Zur Vermeidung metallischer Gegenstände im Wirkungsbereich der Antenne wurde zur Montage ein Befestigungsrahmen aus Holz verwendet. 
Nach der Montage und Ausrichtung der Antenne wurde zuerst die Basisantenne auf die herrschenden Umgebungseinflüsse abgeglichen. Hierfür werden alle manuellen Einstellregler zunächst auf ihre Standardwerte justiert und der automatische Abgleichmodus der Antennenanlage aktiviert. Dieser Vorgang wird nun bei der Ergänzungsantenne wiederholt.

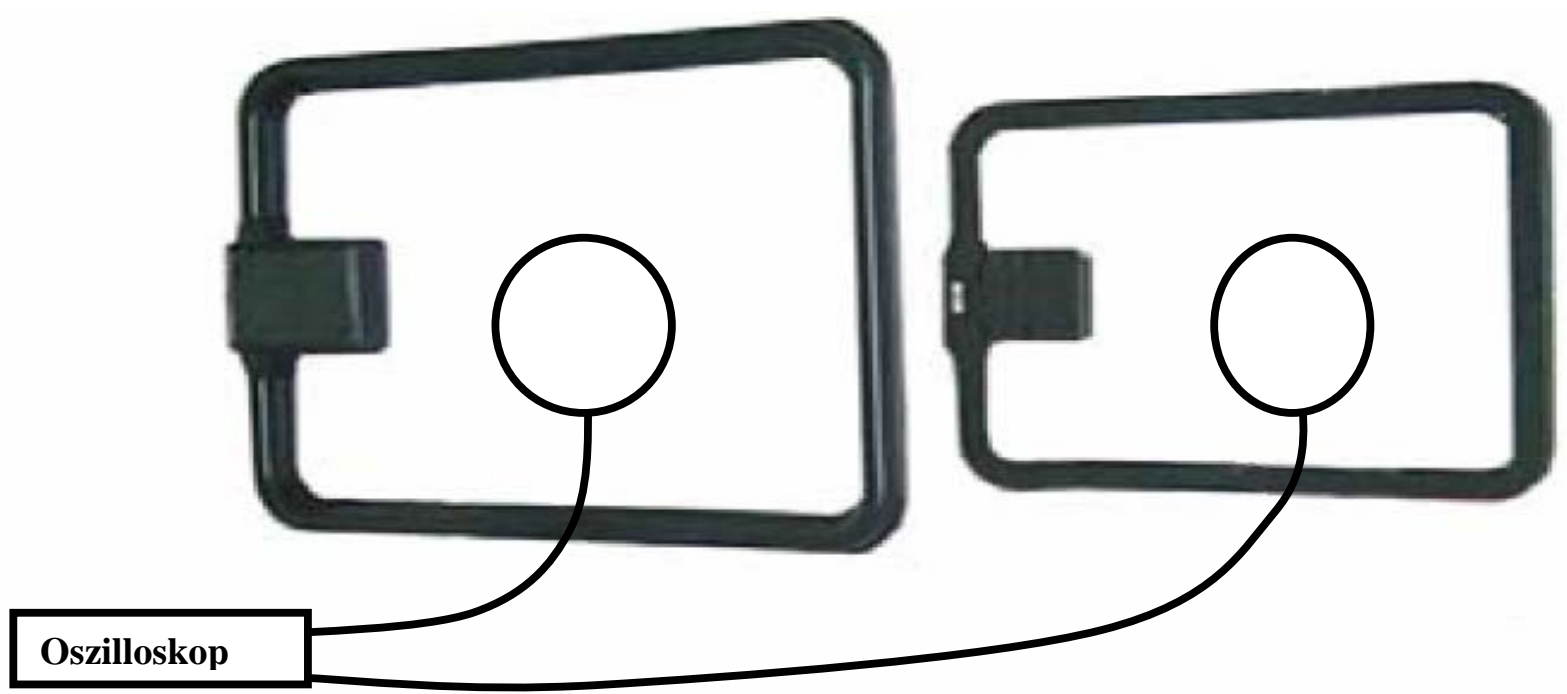

Abb. 4: Basis- und Ergänzungsantenne mit prinzipiellem Messaufbau zur Signalabgleichung

Nun werden die Signale beider Antennen aufeinander abgeglichen. Hierzu werden, wie in Abb. 4 gezeigt, zwei Messantennen mittig in die Antennenringe gehalten. Die Messsignale werden mittels eines Oszilloskops visualisiert. In Abb. 5 sind beispielhaft die Signalströme der Basis- und der Ergänzungsantenne zu sehen. Um einen zuverlässigen Betrieb der Anlage zu gewährleisten darf die Amplitudendifferenz max. 30\% betragen. Der Phasenwinkel, also der zeitliche Versatz der Signale, sollte bei $90^{\circ}+/-10^{\circ}$ (Herstellerangabe) liegen. Abb. 5 zeigt den Abgleich der Antennen des Versuchsstandes. Die Amplitudendifferenz ist minimal, und der Phasenwinkel beträgt $90^{\circ}$. Erste Tests haben gezeigt, das es wichtig ist diese Werte möglichst exakt einzuhalten.

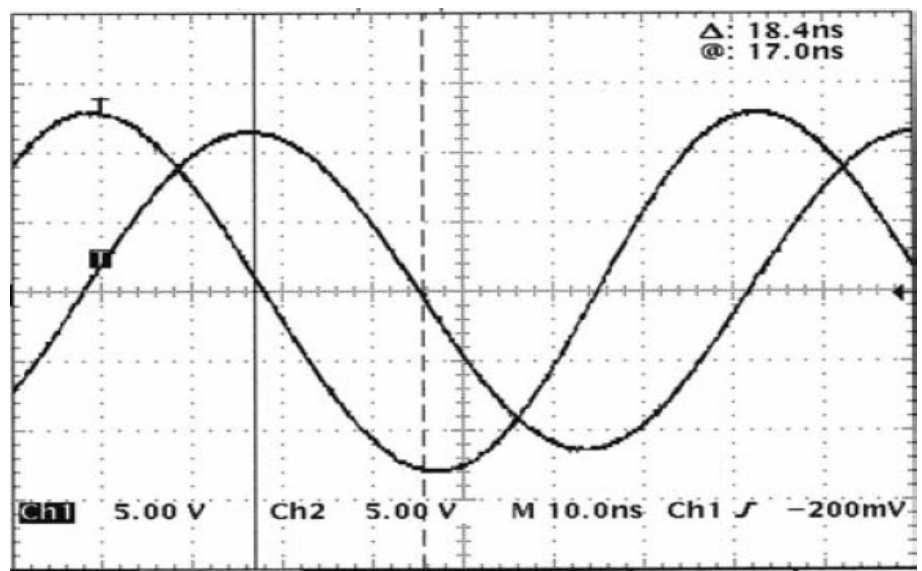

Abb. 5: Gemessenes Signal der Antennenanlage

\subsection{Vorversuche}

Die soeben vorgestellten Versuchsaufbauten gingen aus Vorversuchen hervor. Es wurde zuerst die rechnerisch ermittelte Leistungsfähigkeit der Anlage überprüft und die Softwareparameter der Anlage auf die maximal lesbare Transponderzahl optimiert.

Die Softwareparameter, wie z.B. Time-Slot-Einstellungen können entsprechend der zu erfassenden Transponderzahl bzw. der Datenmenge optimiert werden. Hierfür wurde ein Versuchsaufbau (s. Abb. 6) mit in einer Ebene liegenden Transpondern verwendet. 


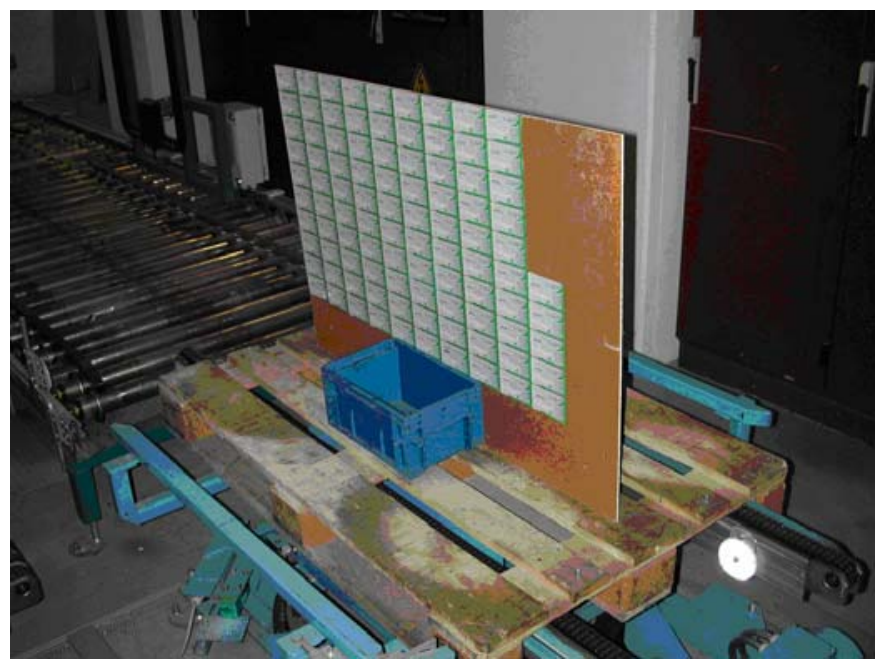

Abb. 6: Zweidimensionaler Vorversuchsaufbau mit 95 Transpondern

Zur Optimierung der Anlage sind die beiden folgenden Parameter nacheinander variiert worden (Ergänzungen zur Anlagenoptimierung: siehe Anhang):

- Time-Slot-Anzahl in der Antikollisionsbehandlung

Von einer Transponder-Kollision spricht man, wenn sich mehrere Transponder gleichzeitig auf eine Anfrage des Readers melden. Bei einer Kollision können die Kontaktversuche getriggert werden, d.h. die Kontaktaufnahme erfolgt in mehreren aufeinander folgenden zeitlichen Abschnitten, bis keine Kollision mehr auftritt. Die Anzahl dieser Abschnitte (Time-Slots) kann erhöht werden.

- Die Anzahl der Re-Readversuche während eines Lesezyklus entspricht der Wiederholhäufigkeit der Kontaktversuche bei Abbruch des Signalkontakts. Bricht während der Kommunikation zwischen Reader und Transponder die Verbindung ab, kann die Anzahl der Versuche zur wiederholten Kontaktaufnahme eingestellt werden.

Die Optimierung der Parameter zeigte, dass die Variation der Time-Slot Einstellungen eine Verbesserung der Erkennungsrate der Transponder zur Folge hatte. Die Erhöhung der Re-Readversuche brachte keine messbare Veränderung. Durch die Optimierung der Softwareparameter konnte die Erkennungsrate bei Verwendung der zweidimensionalen Anordnung mit 95 Transpondern von 99,53\% erreicht werden (entsprechend Abb. 6).

Nach Abschluss des Vorversuches mit zweidimensionaler Transponderanordnung wurden erste Tests mit dem geplanten KLT-Aufbau mit 96 Transpondern, wie in Abb. 7 dargestellt, getätigt. Hier waren zwölf KLT in drei Lagen aufeinander gestapelt. Je KLT waren acht Transponder befestigt, also zwei je Seite. Es wurde versucht mit möglichst vielen Transpondern zu arbeiten, da die Anlage an ihrer rechnerisch ermittelten Leistungsgrenze betrieben werde sollte. Allerdings konnten hier trotz nochmaliger Überprüfung der Softwareparameter nur in 86,98\% der Lesezyklen alle 96 Transponder erkannt werden. Bei allen anderen Lesezyklen wurde mindestens ein Transponder nicht erkannt. Es stellt sich nun die Frage, warum die Erkennungsraten des KLT-Aufbau deutlich unter der zweidimensionalen Anordnung lag (Ergänzungen: siehe Anhang).

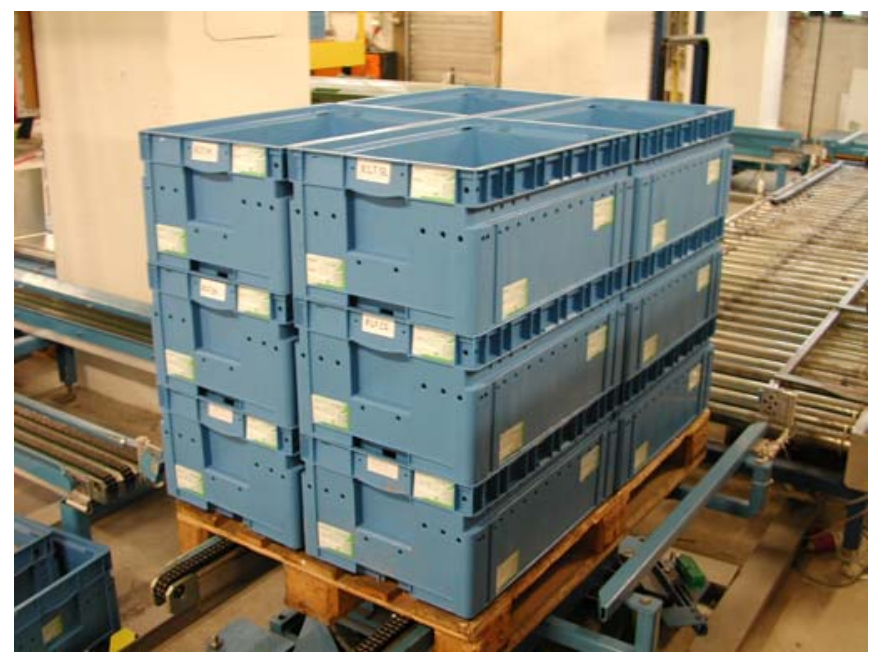

Abb. 7: KLT-Aufbau mit 96 Transponder (Acht je KLT) 
Tests mit Variationen des Versuchsaufbaus, z.B. das Ersetzen einzelner Stapellagen durch KLT ohne Transponder und das Durchtauschen der Stapellagen von oben nach unten, ergaben, dass weder die KLT an sich einen negativen Einfluss auf die Erkennungsrate haben, noch dass Transponder beschädigt waren.

Für die Verschlechterung der Erkennungsraten der Transponder bei Wechsel von der zweidimensionalen Anordnung zum KLT-Versuchsaufbau ist demnach die nun dreidimensionale Anordnung verantwortlich. Die Transponder des in Abb. 6 vorgestellten Versuchsaufbaus sind alle in einer Ebene mit immer gleichem Abstand zur Antenne angebracht und besitzen alle die gleiche räumliche Ausrichtung. Der KLT-Aufbau (nach Abb. 7) weist unterschiedliche räumliche Transponderausrichtungen auf. Die Transponder sind auf den Längs- und Querseiten der KLT angebracht. Durch die Verteilungen in allen drei Raumrichtungen sind auch die Abstände der jeweiligen Transponder zur Antenne unterschiedlich.

Infolgedessen musste nun die Transponderzahl je KLT auf ein vernünftiges, sicher noch zu handhabendes Maß reduziert werden. Aus Gründen der Symmetrie des Aufbaus und der praxisnahen Gestaltung der Ladeeinheiten wurde die Anzahl der Transponder je KLT von acht auf vier reduziert. Es ist nun noch ein anstatt zwei Transponder je KLT-Seite montiert, pro Palette insgesamt 48 Stück.

In der folgenden Abbildung sind die Erkennungsraten der zweidimensionalen Anordnung, des KLT-Aufbaus mit 96 Transpondern sowie des letztendlich verwendeten KLT-Versuchsaufbaus mit 48 Transpondern zu sehen. Die Diagramme zeigen die prozentuale Verteilung der je Lesung erkannten Transponderanzahl. Man erkennt, dass bei den Plattenversuchen mit zweidimensionaler Transponderanordnung in 99,53\% aller Lesungen alle 95 Transponder erkannt worden sind, in den restlichen 0,47\% der Lesungen jeweils zwischen 94 und 91 Transponder. Im Vergleich hierzu fällt beim KLT-Aufbau mit 96 Transpondern die Anzahl der Lesungen, in denen alle Transponder erkannt werden, auf 86,98\% ab, in nur 11,43 \% der Lesungen werden noch 95 Transponder erkannt.

Bei dem letztendlich verwendeten KLT-Versuchsaufbau mit 48 Transpondern steigt die Anzahl der Lesungen mit allen erkannten Transpondern wieder auf 99,47 \%, wobei die Mindestanzahl der erkannten Transponder je Zyklus auch auf 47 gestiegen ist.

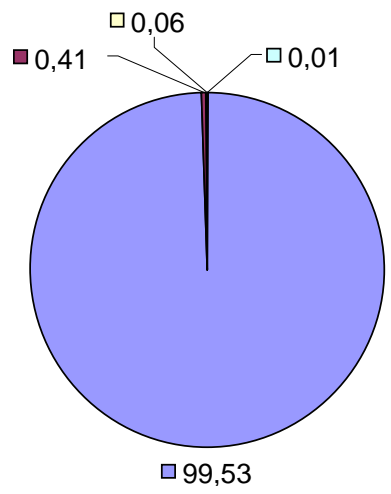

Plattenversuche mit 95 Transpondern
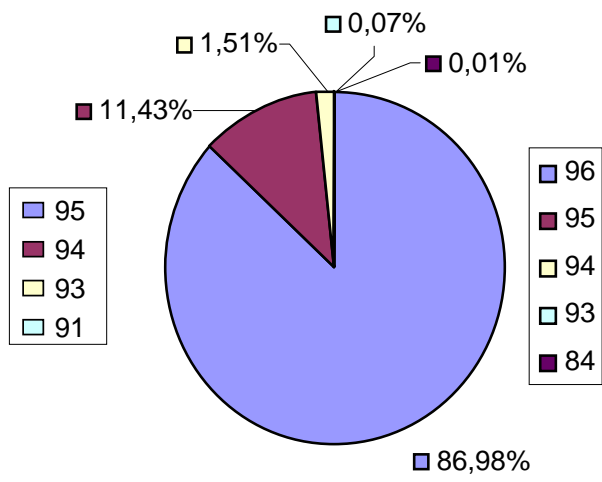

$\square 86,98 \%$

KLT Aufbau, leer, 96 Transponder

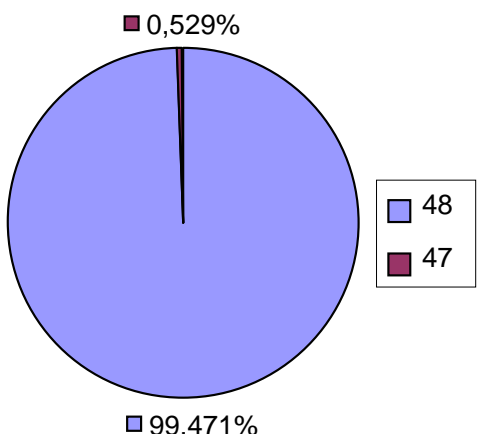

KLT Aufbau, leer, 48 Transponder

Erklärung, Bsp. linkes Diagramm: In 99,53 \% der Lesezyklen wurden alle 95 Transponder erkannt

Abb. 8: Erzielbare Erkennungsraten bei jeweils optimierter Parametereinstellung mit den jeweiligen Versuchsaufbauten

\subsection{Ziele der Reihenversuche}

Mittels der nun gefundenen Anordnungen lassen sich Aussagen über die Erkennungsraten der einzelnen Transponder in Abhängigkeit ihrer Position am Versuchaufbau machen.

Die Ziele der Versuche sind:

- Ermittlung des Einflusses von Füllstoffen auf das Identifikationsverhalten der Transponder

- Ermittlung der Erkennungsraten auf KLT-Basis mit verschiedenen Füllstoffen. Es wird die Frage untersucht, wie eine 100 \%-ige Lesbarkeit eines KLT (stellvertretend für eine Ladeeinheit) umsetzbar ist

- $\quad$ Ermittlung der optimalen Transponderpositionen an den KLT

- Ableiten von generellen Regeln, die bei Implementierung von RFID-Technik zu beachten sind 


\subsection{Durchführung der Reihenversuche}

Bei den Versuchsreihen wird jeweils die Ein-Eindeutige ID-Nummer der Transponder ausgelesen. Sie wird je Transponder nur einmal vergeben und ist irreversibel auf dem Transponder gespeichert. Die Position eines jeden Transponders ist dokumentiert, womit eine Zuordnung der Erkennungsrate für jede Transponderposition möglich ist.

Es wird nacheinander der Einfluss unterschiedlicher Füllmaterialien auf das Identifikationsverhalten der Transponder untersucht:

- Stahlbleche

- Aluminiumbleche

- $\quad$ PET-Wasserflaschen

- Pressspanplatten

- MDF-Platten (Mittel-Dichte-Faserplatten)

- Massivholzbretter

- Sperrholz

- u.a.

Die Ergebnisse für die Metallwerkstoffe liegen bereits vollständig vor und werden im Nachfolgenden vorgestellt. Die Versuche für die anderen Füllstoffe laufen noch und müssen noch ausgewertet werden.

\subsection{Ergebnisse der Versuche}

\section{Ungefüllte KLT:}

Bei Versuchen mit leeren KLT zeigen beide Versuchsaufbauvarianten A und B durchweg sehr gute Erkennungsraten der einzelnen Transponder. Bei Aufbauvariante A werden 46 von 48 Transpondern immer erkannt, lediglich zwei der Transponder werden je einmal während der Versuchsdauer (9026 Lesezyklen) nicht gelesen. Ähnliches lässt sich hier auch für die Variante B sagen, wo nur drei Transponder je Versuch einmal nicht gelesen wurden (3977 Lesezyklen).

\section{Einsatz metallischer Füllstoffe:}

Sie gelten beim Einsatz von RFID aufgrund ihrer abschirmenden Wirkung als problematisch. Die Versuche des IFT haben zum einen diese Tatsache bestätigt, aber auch gezeigt, dass Ladeeinheiten mit metallischen Füllstoffen durchaus gelesen werden können, wenn bestimmte Randbedingungen beachtet werden. Dies ist nicht zuletzt von der Lage und der Ausrichtung der Transponder auf der Ladeeinheit abhängig.

Bei Stahl- und Aluminiumblechen variiert die Erkennungsrate stark in Abhängigkeit von der Position der einzelnen Transponder innerhalb der Versuchsaufbauten.

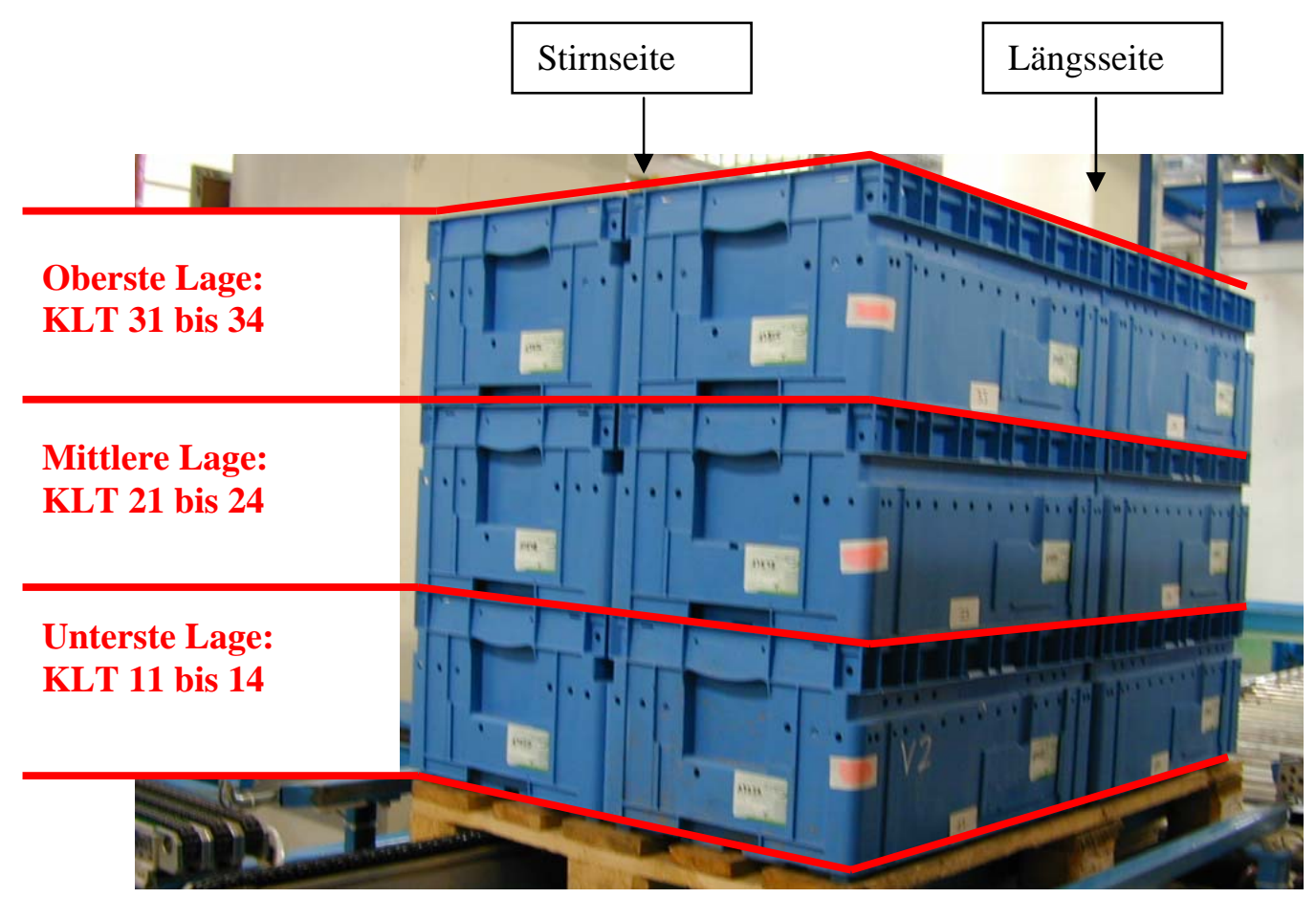

Abb. 9: Lagenstapelung der KLT auf Palette und Nummerierung der KLT 
Allerdings sind große Unterschiede je nach Position der Transponder in den einzelnen Stapellagen zu erkennen. In Abb. 10 ist beispielhaft die Verteilung der Erkennungsraten der einzelnen Stapellagen bei Befüllung mit Stahlblechen für die Versuchaufbauvarianten A und B gemäß Abbildung 3 abgebildet. Links ist die Variante A und rechts die Variante B zu sehen, wobei jeweils als Draufsicht schematisch die einzelnen Stapellagen (Unterste, mittlere und oberste Stapellage) der Versuchsaufbauten mit den jeweiligen KLT gezeigt sind. Die farbigen Markierungen symbolisieren jeweils einen Transponder entsprechend der Lage an den Versuchsaufbauten.
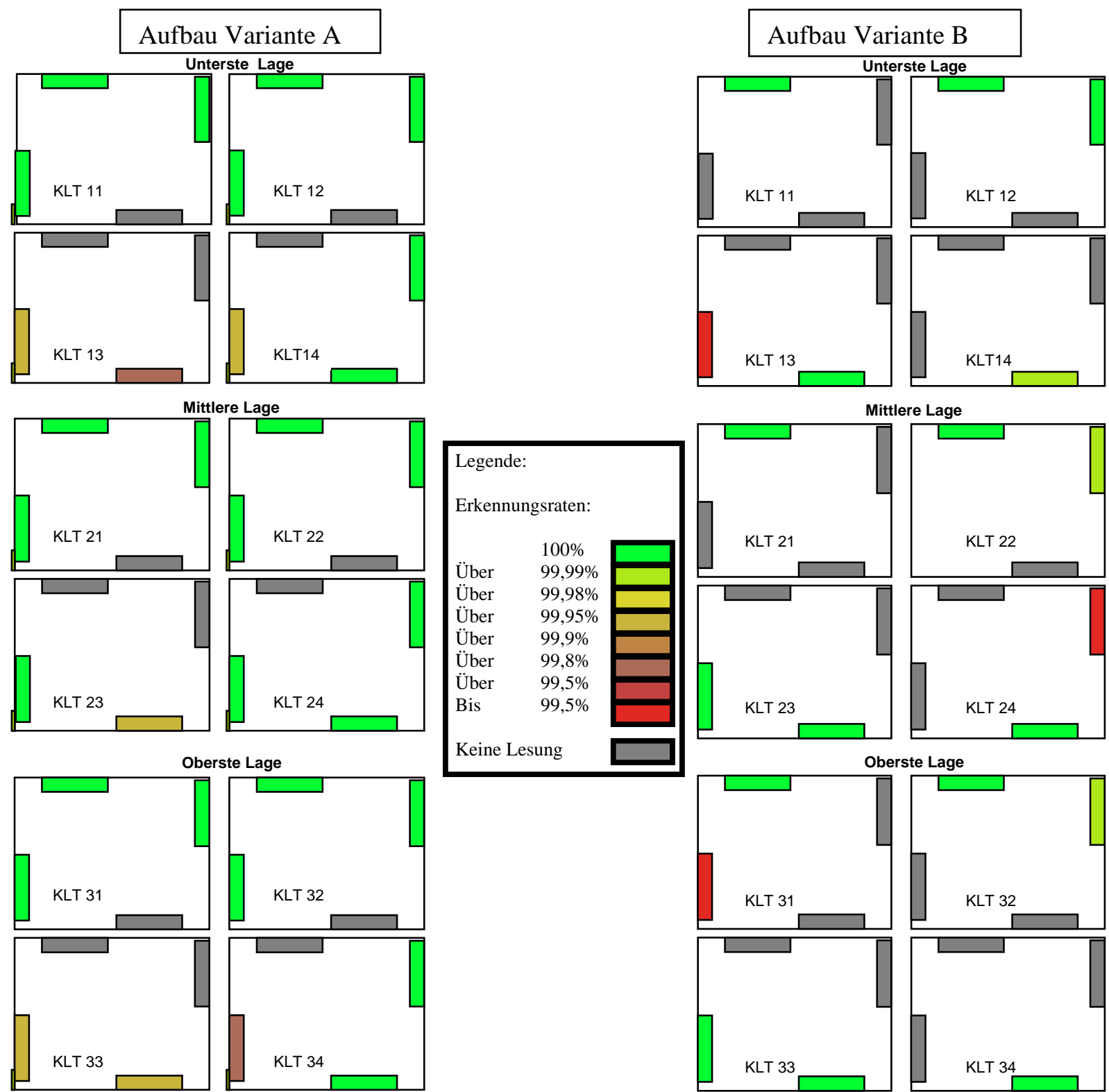

Abb. 10: Erkennungsraten der einzelnen Transponder

Es ist zu erkennen, dass in der Vertikalen, also jeweils von der untersten bis zur obersten Stapellage, keine systematischen Abweichungen in den Erkennungsraten vorhanden sind. Allerdings ist feststellbar, dass die Transponder, die auf den innen liegenden Längsseiten der KLT angebracht sind, bei beiden Versuchsaufbauten, wie zu erwarten, nicht erkannt werden.

Ein großer Unterschied zwischen beiden Aufbauvarianten besteht allerdings bei den Transpondern an den innen liegenden KLT-Stirnseiten. Bei Variante A werden die Transponder an den inneren KLT-Stirnseiten relativ gut erkannt, bei Variante B nicht.

Wie in Abb. 10 zu sehen, werden Transponder an den äußeren Längsseiten der Versuchaufbauten relativ problemlos bei beiden Versuchsaufbauten erkannt. Sobald sich die Transponder vollflächig hinter oder zwischen den Metallen verbergen, ist eine Lesung so gut wie ausgeschlossen. Dass im Inneren des Versuchsaufbaus A trotz der Metalle Transponder erkannt wurden, liegt an ihrer Positionierung. Wie oben beschrieben, sind die stirnseitigen Transponder in der Nähe der KLT-Oberkante positioniert, sie werden also nicht vollflächig über- 
deckt. Diesem Umstand ist es zuzuschreiben, dass diese Transponder trotz Metallfüllung erkannt werden können.

Sollen also Behälter mit metallischen Inhaltsstoffen per RFID identifiziert werden, steigt die Zuverlässigkeit des Identifikationsvorganges beträchtlich, wenn man Transponder generell nicht im Inneren der Ladeeinheit, also nicht hinter metallischen Flächen, anbringt.

Wenn aus technischen Gründen diese definierte Ausrichtung der Ladeeinheit nicht möglich ist, bieten sich hier zwei mögliche Alternativen an:

1. Die Belabelung mit zwei Transpondern, die sich diagonal versetzt auf sich gegenüberliegen Seiten der Ladeeinheit befinden. Somit ist sichergestellt, dass immer ein Transponder nach außen in Richtung Antenne zeigt. Nachteilige ist, dass zur Identifikation die Ein- Eindeutige ID-Nummer des Transponders nicht genutzt werden kann, da es für eine Ladeeinheit immer zwei „Zwillingstransponder“ gibt, die bei einer Identifikation einander zugeordnet werden müssen.

\section{2 „Zwillingstransponder“ je KLT}

KL

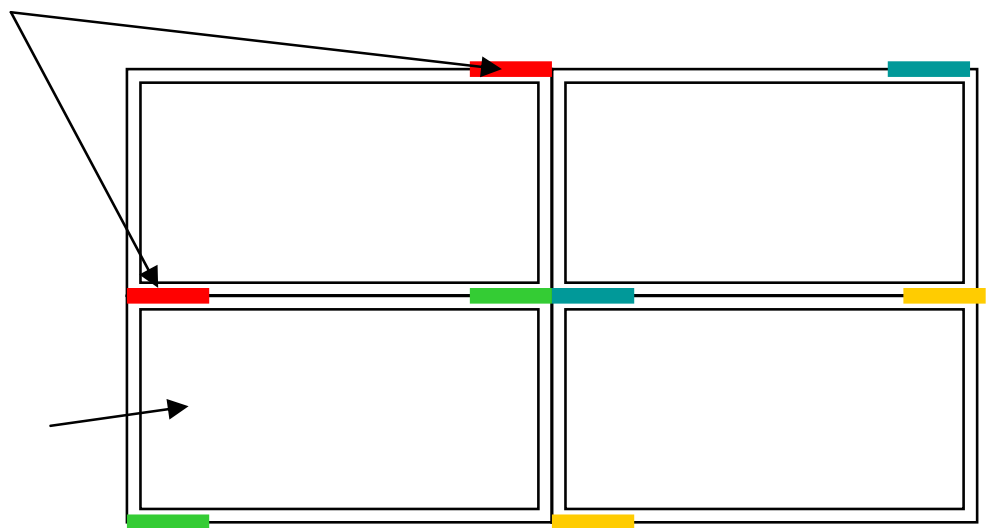

Abbildung 11: KLT mit je zwei Transpondern (Draufsicht)

2. Wenn Ladeeinheiten nicht bis zu ihrer Oberkante gefüllt werden können (Deckel, Eintauchreserve für obere Ladeinheit,...), sollte der Transponder möglichst in diesem Bereich angebracht sein. Dort sind die Transponder nicht direkt metallisch abgeschirmt und die Transponder prinzipiell lesbar. Allerdings müsste dies für jede Art Aufbau geprüft werden, ob das sich in diesem „Spalt“ ausbreitende Feld für eine Identifizierung ausreichend ist.

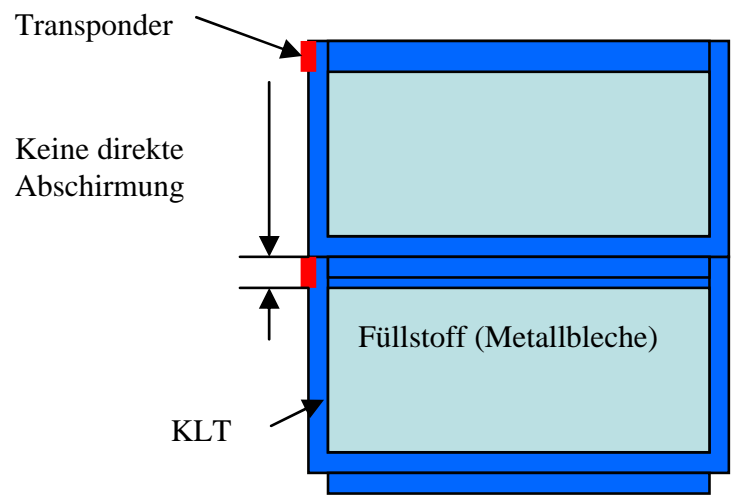

Abb. 12: Anbringung der Transponder außerhalb der nutzbaren Ladehöhe

\section{Forschungsbedarf}

In den letzten Jahren wurden theoretische Erkenntnisse über Abstrahlcharakteristiken, Lesefelder und dem Einfluss verschiedener Medien unter Laborbedingungen von RFID-Technik untersucht, in der Regel unter abgeschirmten, genau definierbaren Umgebungsparametern.

Heutige Forschungs- und Entwicklungsprojekte haben den Einsatz von RFID innerhalb der Supply-Chain und deren Optimierung als Schwerpunkt.

Bisher kaum oder nur ansatzweise behandelt ist allerdings die technische Schnittstelle zwischen Transponder und Schreib-Lesestation. Eine systematische Untersuchung, wie und unter welchen Randbedingungen sich 
Transponder im industriellen Umfeld, einzeln wie auch im Pulk, zuverlässig auslesen lassen, fehlt nach Meinung des IFT bis heute. Viele RFID-Szenarien basieren auf der Funktionalität und der Zuverlässigkeit der RFID Systeme, ohne abgesicherte Daten über die technische Zuverlässigkeit dieser Systeme in der Praxis zu haben.

Die ersten Untersuchungen des IFT zeigten, dass die theoretische und berechnete Funktionalität und Zuverlässigkeit des Identifikationsvorganges von den realen Ergebnissen unter realitätsnahen Bedingungen erheblich abweichen kann.

Daher ist es, trotz theoretischer Vorüberlegungen, unabdingbar, Transponder, Ladeeinheit, Fördertechnik und die Schreib-Lesestation aufeinander abzustimmen. Diese Abstimmung und Überprüfung muss für jeden Einzelfall vorgenommen werden, da beispielsweise schon die Variation des Anbringungsortes bei sonst gleicher Ladeeinheit erhebliche Unterschiede der Erkennungsrate zur Folge haben kann.

Daher ist es außerordentlich wichtig, die Schnittstelle „Transponder und Schreib-/Lesestation“ auch unter praxisnahen Bedingungen mit realen förder- und lagertechnischen Komponenten weiter zu untersuchen, da der Nutzen des RFID- Einsatzes neben der Standardisierung maßgeblich von der Zuverlässigkeit der Identifikationsvorgänge abhängt.

Es sind Fragen zu klären, wie man eine Ladeeinheit optimal belabelt und welche Randbedingungen bei der Benutzung von RFID beachtet werden müssen. Ziel der Untersuchungen sollen Empfehlungen sein, unter welchen technischen Bedingungen der Identifikationsvorgang optimal gestaltet werden kann. Die Versuche am IFT hierzu werden deshalb fortgesetzt und zusätzlich weitere Versuche mit passiven $868 \mathrm{MHz}$-Transpondern unter gleichen Bedingungen durchgeführt, um Vergleiche mit der 13,56 MHz-Technik ziehen zu können.

\section{Literatur}

[tenHompel04]

[Finkenzeller02]

ten Hompel, Michael (Herausgeber); Lange, Volker (Herausgeber): RFID - Logistiktrends für Industrie und Handel, Verlag Praxiswissen GmbH, Dortmund, 2004

Finkenzeller, Klaus: RFID Handbuch - Grundlagen und praktische Anwendungen induktiver Funkanlagen, Transponder und kontaktloser Chipkarten, Hanser Verlag München, Wien, 2002

[Friess00]

Friess, G.: Beitrag zur Integration von Handelsdaten, Stuttgart, Universität, Fakultät konstruktions- und Fertigungstechnik, IFT, Diss., 2000

[Roos02] Roos, H.J.; Coskun, S.: Standardisierte RFID Systeme in der Automobilindustrie, Institut für Fördertechnik und Logistik, Universität Stuttgart, Abschlussbericht, Mai 2002

[GS10.J.]

[Jansen05]

[Bapat04]

GS1 Germany GmbH, Maarweg 133, 50825 Köln, http://www.epcglobal.de

Jansen, R.; Hustadt, J.: Stand der der Technik und Entwicklungspotential, In: Hebezeuge und Fördermittel, 45 (2005) 3, S. 112-113

Bapat, V.; Tinnell, K.: RFID in Manufacturing - A practical guide on extracting measurable value from RFID implementations in plant and warehouse operations, White Paper, Rockwell Automation, 2004

[Overmeyer05] Overmeyer, L.; Vogeler, S.: RFID - Grundlagen und Potentiale, Logistics Journal, ISSN 1860-5923, http://www.elogistics-journal.de, 2005, DOI 10.2195/LJNot_Ref_d_Overmeyer_032005

\section{Anhang}

Im Rahmen von Vorversuchen zur Ermittlung eines geeigneten Versuchaufbaus und zur Optimierung der Anlagenparameter wurden Tests ohne Füllmaterialien durchgeführt. Diese haben gezeigt, dass die Anzahl der zuverlässig lesbaren Transponder von zwei Faktoren abhängig ist:

\subsection{Anordnung und Ausrichtung der Transponder im Raum}

Bei gleicher Einstellung der Anlagenparameter (Time-Slot-Anzahl und Re-Read-Versuche) liegt die Zahl zuverlässig erkennbarer Transponder in zweidimensionaler Anordnung auf einer Ebene (siehe Vorversuche) deutlich über der dreidimensionalen Anordnung des KLT-Aufbaus (ungefüllt). In folgender Abbildung sind exemplarisch die Erkennungsraten bei gleicher Parametereinstellung mit dem Plattenaufbau und dem KLT-Aufbau mit 96 Transpondern zu sehen. Im Falle des zweidimensionalen Aufbaus werden in 98,92 \% der Lesezyklen alle 100 Transponder erkannt, im Falle des KLT-Aufbaus wurden lediglich in 62,95\% der Lesezyklen alle 96 erkannt. 

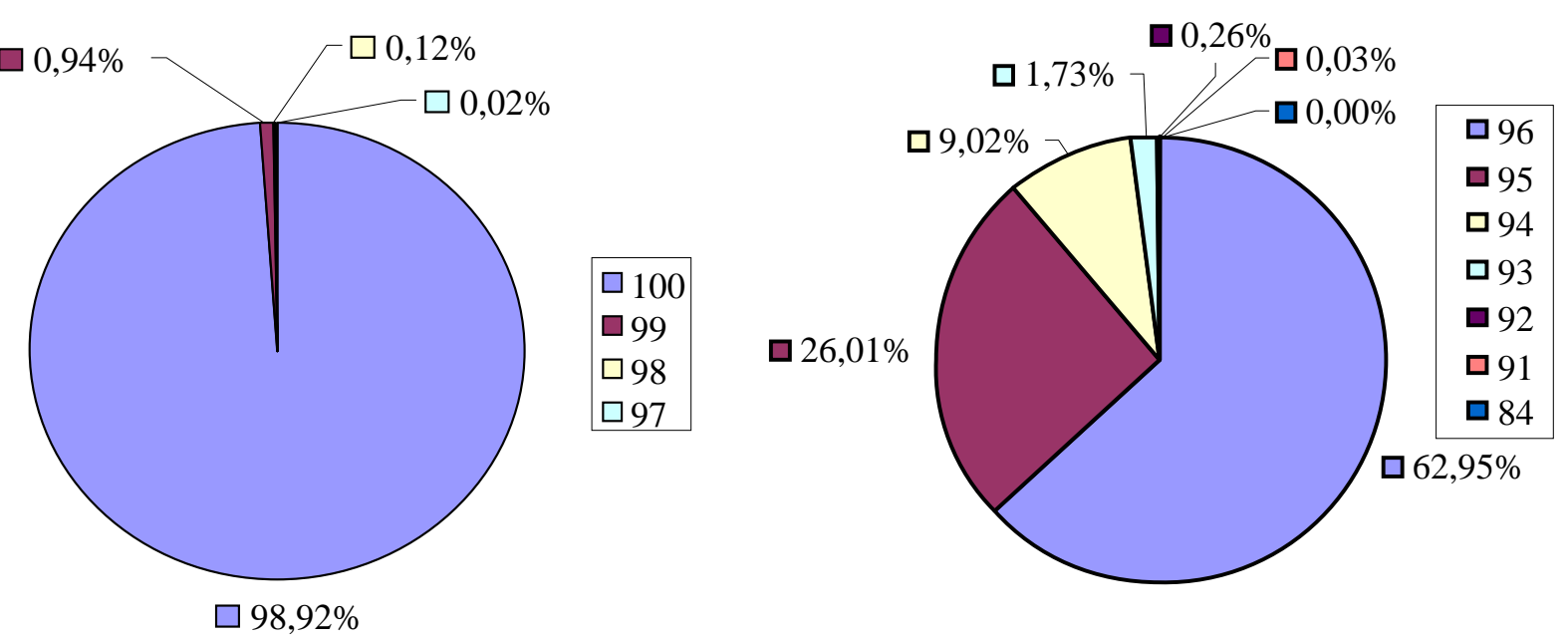

Abb. 13: Erkennungsrate von zweidimensionalem Plattenaufbau (100 Transponder, links) und dreidimensionalem KLTAufbau (96 Transponder, rechts) bei gleicher Parameter- Einstellung (Beispiel)

Es ist also offensichtlich, dass die mögliche Anzahl der Transponder maßgeblich von deren Anordnung im Raum abhängig ist. Bei einer möglichst „,idealen“ räumlichen Anordnung, wie bei den Plattenversuchen (Abb. 14), ist die erreichbare Transponderzahl höher als bei dreidimensionalen Anordnungen mit unterschiedlichen Ausrichtungen im Raum.

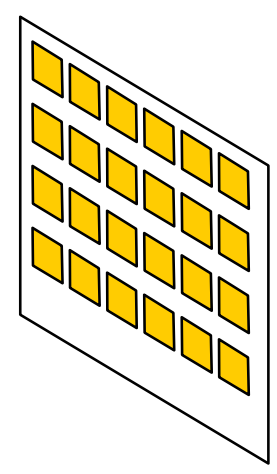

Zweidimensionale Anordnung in einer Ebene, 95 Transponder

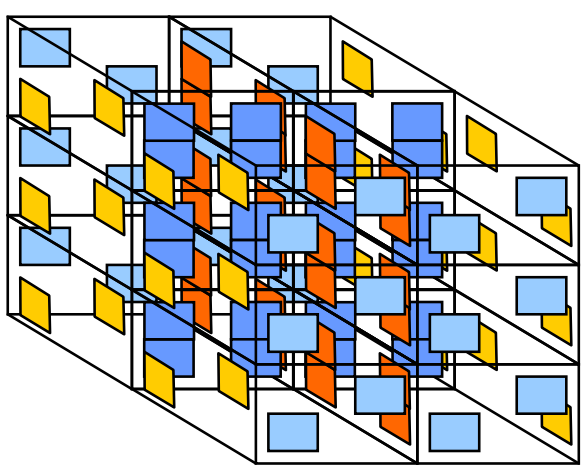

Dreidimensionale Anordnung im Raum mit unterschiedlicher Ausrichtung der Transponder, 96 Transponder

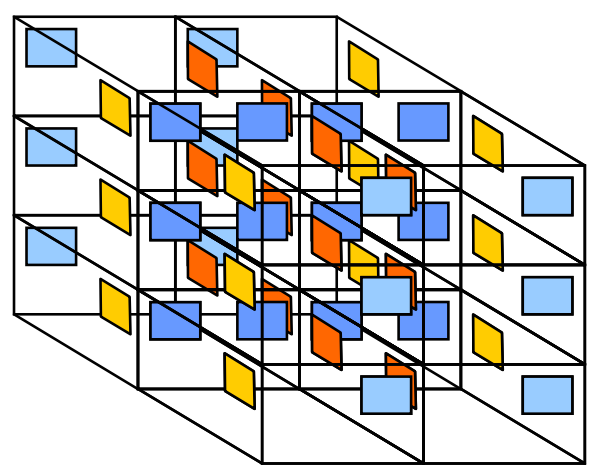

Dreidimensionale Anordnung im Raum mit unterschiedlicher Ausrichtung der Transponder, 48 Transponder

Abb. 14: Räumliche Anordnung der Transponder bei den Plattenversuchen sowie den KLT-Aufbauten mit 96 und 48 Transpondern

Bedingt durch die Anbringung der Transponder auf der Versuchplatte haben alle Transponder nahezu die gleiche Ausrichtung und Lage im Antennenfeld bei dessen Durchquerung. Es gelten also für alle Transponder beinahe die gleichen Lesebedingungen, wodurch alle Transponder qualitativ gleichwertig im Antennenfeld erfasst werden konnten.

Bei den KLT-Aufbauten hingegen befinden sich die Transponder in mehreren Ebenen. Zusätzlich haben Sie unterschiedliche Ausrichtungen zum Antennenfeld, d.h. der Eintrittswinkel und der Abstand zum Antennenfeld sind unterschiedlich. Dadurch sind die Transponder bei Eintritt und Durchquerung des Antennenfeldes in unterschiedlichen Zonen des Antennenfeldes unterschiedlich gut lesbar. In Abb. 15 ist die ideale Ausrichtung eines Transponders mit Spulenantenne in Relation zum Antennenfeld, bzw. dessen Feldlinien schematisch angedeutet. Auch der Verlauf der Feldlinien innerhalb einer Gate-Antenne ist überall parallel. So entstehen für Transponder, je nach ihrer räumlichen Ausrichtung, Zonen besserer und schlechter Lesbarkeit. Beispielsweise ist der Transponder am besten zu lesen, wenn er senkrecht zum Antennenfeld steht, da er so die meiste Energie aus diesem Feld ziehen und damit auch am besten senden kann. 


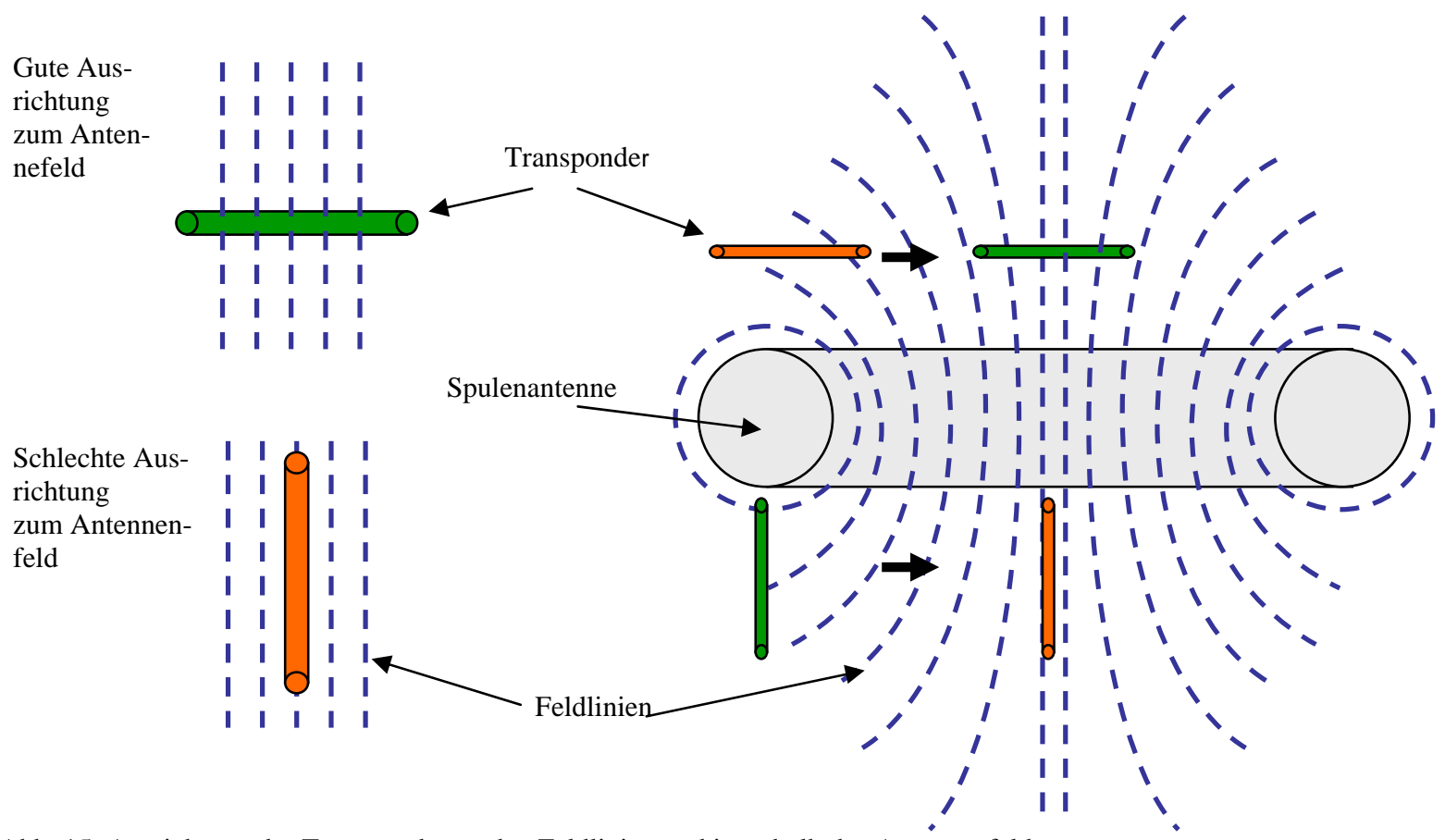

Abb. 15: Ausrichtung der Transponder zu den Feldlinien und innerhalb des Antennenfeldes

\subsection{Optimierung der Anlagenparameter}

Einen entscheidenden Einfluss auf die Lesbarkeit der Transponder hat eine optimal auf die Ladeeinheit abgestimmte Anlagenkonfiguration. Im Folgenden sind zwei Diagramme zu sehen, welche die Erkennungsrate des KLT-Versuchsaufbaus mit 96 Transpondern bei verschiedenen Einstellungen der Time-Slot-Anzahl zeigen:

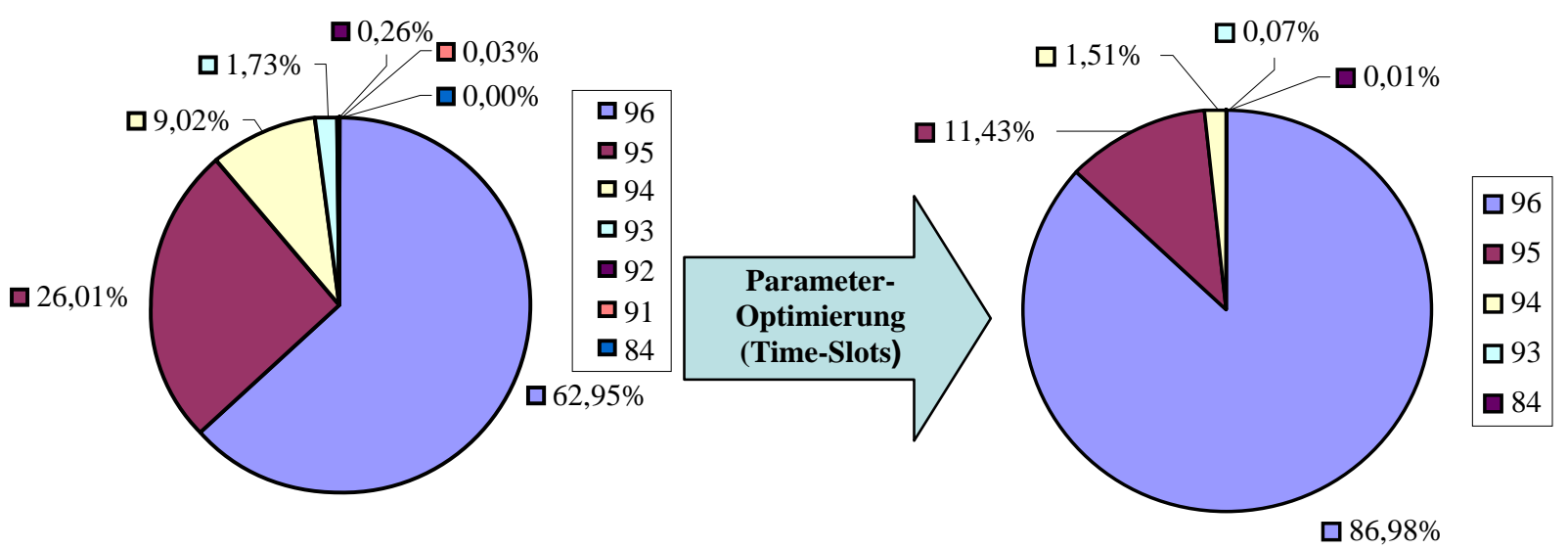

Abb. 16: Erhöhung der Erkennungsrate durch Parameteroptimierung bei KLT-Vorversuchsaufbau mit 96 Transpondern (Beispiel)

Für die optimale Performance einer Anlage ist es also wichtig, diese im Vorfeld durch Versuche auf die geplante Anzahl Transponder je Lesevorgang hin zu optimieren. 